\title{
Streptomycin Resistance and Morphological Variation in Phytophthora cactorum
}

\author{
By D. S. SHAW* AND C. G. ELLIOTT \\ Botany Department, University of Glasgow \\ (Accepted for publication I I September 1967)
}

\begin{abstract}
SUMMARY
Strains of Phytophthora cactorum resistant to streptomycin were obtained by incubating zoospores in liquid medium containing the drug. One of the strains required streptomycin for growth. In this dependent strain and one of the resistant strains, the dependence or resistance was transmitted to both asexual and selfed sexual progeny without any segregation for sensitivity. Change from dependence to resistance occurred with low frequency. Some of the resistant and the dependent strains showed much greater morphological variation than the wild type. Continuing segregation for morphological characters was observed during vegetative growth and in the asexual progeny of single zoospores, and the ability to segregate was not lost following sexual reproduction (selfing). Cultures were more variable after storage at $3^{\circ}$. There was evidence suggesting a particulate nature for the determinants of these morphological differences, normal zoospores having one such determinant.
\end{abstract}

\section{INTRODUCTION}

Virtually nothing is known of the genetics of any of the oomycetes, among them the genus Phytophthora, which includes many important plant pathogens. The most obvious reason for the neglect of these fungi by geneticists is the difficulty in germinating oospores and so of obtaining sexual progeny. Hence not only do we not know the basis of the interesting examples of variation in morphology and pathogenicity and of the complex mating behaviour which have been described in various phytophthoras; we do not even know for certain at what point in the life history meiosis occurs, and whether the somatic hyphae are haploid or diploid. Recent cytological evidence from species of the genera Pythium, Phytophthora and Achlya (Sansome, 196I, 1963, 1965) would seem to indicate that meiotic divisions take place in the gametangia just before fertilization. If this were so the life-cycle in these species and possibly in all oomycetes would be based on the diplontic pattern. As interpretation of division figures in these fungi is extremely difficult, genetical proof of this hypothesis is necessary. Mullins \& Raper (I965) obtained data on the inheritance of maleness and femaleness in Achlya and Dictyuchus, which they interpreted as indicating that the somatic hyphae are diploid. However, these characters are complex, and a study by using simple gene markers is desirable.

Attempts to obtain 'biochemical' mutants of Phytophthora (e.g. Buddenhagen, I958; Clarke \& Robertson, I966) have not been successful. This might be due to technical matters; but alternatively, if these fungi are diploid, as Sansome suggested,

* Present address: School of Plant Biology, University College of North Wales, Bangor. 
the lack of success would be understandable, as such mutants in Aspergillus and yeast are always recessive, and so would not be expected to reveal themselves until after recombination. On the other hand, mutations to drug resistance have often been found to be dominant or semidominant (Roper \& Käfer, 1957; Warr \& Roper, 1965; Middlekauf et al. 1957) and so could be selected directly in a diploid. We therefore set out to select drug-resistant mutants of Phytophthora cactorum (Leb. \& Cohn) Schroet., and this paper reports a study of streptomycin resistance.

Unfortunately we have as yet no genetical information about the resistant strains obtained. We have succeeded in germinating oospores in large numbers (Shaw, 1967), but the fungus is homothallic, and in making a cross one would need to be able to distinguish hybrid sexual progeny from selfed progeny by their showing recombination between markers from the two strains; such markers are not yet available. However, if the strain were diploid and heterozygous for a dominant gene conferring resistance, its selfed progeny would segregate; if the strain were haploid, no segregation would be expected. If the resistance were due to a cytoplasmic condition, either no segregation or non-Mendelian segregation might occur. It is therefore of interest to compare the sexual progeny of the resistant strains, resulting from the germination of oospores, with their asexual progeny, resulting from zoospores.

\section{METHODS}

The strain of Phytophthora cactorum used was IMI 21168 , obtained from the Commonwealth Mycological Institute, Kew. Incubation was at $24^{\circ}$ in the dark.

Standard medium was made up from two separate solutions, which were kept unautoclaved in polythene bottles at $-15^{\circ}$. The first solution contained (g.): sucrose, 10 ; L-asparagine, I.0; $\mathrm{MgSO}_{4} \cdot 7 \mathrm{H}_{2} \mathrm{O}, 0.25 ; \mathrm{KH}_{2} \mathrm{PO}_{4}, 0.5$; thiamin hydrochloride, I mg.; trace element solution, I $\mathrm{ml}$; water, $250 \mathrm{ml}$. (The trace element solution contained $\mathrm{Na}_{2} \mathrm{~B}_{4} \mathrm{O}_{7}$. IOH$_{2} \mathrm{O}, 88 \mathrm{mg}$.; $\mathrm{CuSO}_{4} \cdot 5 \mathrm{H}_{2} \mathrm{O}, 393 \mathrm{mg}$.; $\mathrm{Fe}_{2}\left(\mathrm{SO}_{4}\right)_{3} \cdot 9 \mathrm{H}_{2} \mathrm{O}, 910 \mathrm{mg}$.; $\mathrm{MnCl}_{2}$. ${ }_{4} \mathrm{H}_{2} \mathrm{O}$, $72 \mathrm{mg}$; $\mathrm{Na}_{2} \mathrm{MoO}_{4} \cdot 2 \mathrm{H}_{2} \mathrm{O}$, $50 \mathrm{mg}$; $\mathrm{ZnSO}_{4} \cdot 7 \mathrm{H}_{2} \mathrm{O}$, $4403 \mathrm{mg}$; EDTA, $5 \mathrm{~g}$; water, I 1.). The second solution was made by boiling $75 \mathrm{~g}$. frozen garden peas in $250 \mathrm{ml}$. water and filtering off the solid matter. For use the two solutions were thawed, and $500 \mathrm{ml}$. water added and autoclaved at $120^{\circ}$ for $15 \mathrm{~min}$.

Standard medium agar (SMA): 8.0 g. Oxoid Agar no. 3 was added to I 1. standard medium.

Medium for producing oospores was SMA to which was added I \% (w/v) of an oat extract made by refluxing ground oats in light petroleum for $\mathrm{I} \mathrm{hr}$, filtering, and evaporating the solvent from the filtrate.

Pea meal agar. Frozen garden peas, blended, 300 g.; agar, 8 g.; water, I 1.

Soft non-nutrient agar. Agar, 8 g.; water, I 1.

Sucrose salt solution for suspending zoospores (g.): sucrose, $20 ; \mathrm{NaCl}, 0.215 ; \mathrm{KCl}$, $0.0075 ; \mathrm{Na}_{2} \mathrm{~S}_{2} \mathrm{O}_{3} .5 \mathrm{H}_{2} \mathrm{O}, 0.05 ; \mathrm{CaCl}, 0.012 ;$ in I 1. water.

Streptomycin (as Streptomycin Sulphate, Glaxo) was dissolved in sterile water and added to media after autoclaving.

Production of zoospores. Zoosporangial cultures were prepared from 8-day colonies grown on pea meal agar. Three strips of mycelial mat $(7 \times 0.5 \mathrm{~cm}$.) were cut from the surface of a colony and transferred with a minimum of adhering agar to a $9 \mathrm{~cm}$. Petri dish of soft non-nutrient agar. Zoosporangia were formed in these strip cultures within $72 \mathrm{hr}$. They were induced to discharge zoospores by flooding with $5 \mathrm{ml}$. de-ionized 
water at $15^{\circ}$ and incubation at $15^{\circ}$. To prevent the zoospores bursting soon after discharge, $2.5 \mathrm{ml} .4 \%(\mathrm{w} / \mathrm{v})$ sucrose at $15^{\circ}$ was added $30 \mathrm{~min}$. after the initial flooding with water and a further $2.5 \mathrm{ml}$. at $15 \mathrm{~min}$. later, thus bringing the final sucrose concentration to $2 \%$. The cultures were then removed to room temperature $\left(20-22^{\circ}\right)$ and the zoospores harvested.

High viability (above $50 \%$ ) of zoospores in suspensions of low concentration could only be maintained by adding physiological salts to suspensions in sucrose. Zoospore suspensions were diluted into the sucrose salt solution (see above), and viability was then assured for periods of $2-3 \mathrm{hr}$.

This method of producing zoospores was modified when zoospore progeny of a large number of isolates was required. The fungus was grown on discs of pea meal agar $4 \mathrm{~mm}$. diam. and $8 \mathrm{~mm}$. thick in a moist chamber for $72 \mathrm{hr}$, after which each disc was transferred to a I oz. bottle or culture tube containing about $\mathrm{IO} \mathrm{ml}$. of soft nonnutrient agar. After a further $72 \mathrm{hr}$ of incubation, zoospore suspensions were obtained by flooding with $0.5 \mathrm{ml}$. water at $\mathrm{I} 5^{\circ}$ and subsequently adding two lots of $4 \%$ sucrose each of $0.25 \mathrm{ml}$. as before. By this method the amount of pre-sporulation growth was considerably reduced.

Germination of oospores was as described by Shaw (1967).

Measurement of growth. In many cases colonies on agar medium were too variable in density for their diameters to be a good measure of differences between them in growth. However, the light absorption of a whole colony was considered to be a suitable measure of growth. Ten colonies were grown in a $9 \mathrm{~cm}$. Petri dish, to a size not exceeding $15 \mathrm{~mm}$. diam. A Zeiss photomicrographic apparatus which included a photocell connected to a meter, was set up on a microscope stand with a high-intensity lamp. The condenser, objective and eyepiece of the microscope were removed. The light intensity was adjusted to give full-scale deflection on the meter on an uncolonized part of the dish, and the light absorbed by each colony in turn read off.

\section{RESULTS}

\section{Effect of streptomycin on wild type}

Figure I shows the effect of streptomycin on growth of wild type. From each of 50 colonies resulting from a plating of wild type zoospores on SMA, five inocula about $\mathrm{I} \mathrm{mm}$. cube were cut and transferred to SMA+streptomycin $0,3,7 \cdot 5,20$ and $100 \mu \mathrm{g} . / \mathrm{ml}$. Growth was measured photometrically and the histograms show the amounts of growth, grouped in arbitrary but equal intervals according to light absorption. Growth rate, measured by increase in colony diameter, was decreased to half the control value with streptomycin $7 \mu \mathrm{g} . / \mathrm{ml}$.

Zoospores plated directly on SMA with streptomycin 20 or more $\mu \mathrm{g} . / \mathrm{ml}$. produced germ tubes up to I. $5 \mathrm{~mm}$. long with only a few lateral branches (Fig. 2); further growth was inhibited.

\section{Selection of resistant strains}

Each of four 21 . flasks containing $500 \mathrm{ml}$. liquid standard medium + streptomycin $100 \mu \mathrm{g} . / \mathrm{ml}$. was inoculated with $10^{5}$ zoospores and incubated without shaking or stirring for Io days. Eight growths appeared (not all in the one flask) and were transferred to SMA + streptomycin $100 \mu \mathrm{g}$. $/ \mathrm{ml}$. To test the stability of streptomycin tolerance in the absence of drug and in zoospore progenies, each isolate was treated as follows: 
(i) Plates of pea-meal agar were inoculated to provide mycelium for strip cultures. (ii) Zoospores released from strip cultures were plated on SMA and on SMA + streptomycin $100 \mu \mathrm{g}$. $/ \mathrm{ml}$. (iii) Six spores germinated on SMA and six on SMA + streptomycin were transferred to SMA without streptomycin. (iv) Inocula from each of these twelve cultures were tested on SMA + streptomycin $0,100,250$ and $500 \mu \mathrm{g} . / \mathrm{ml}$.
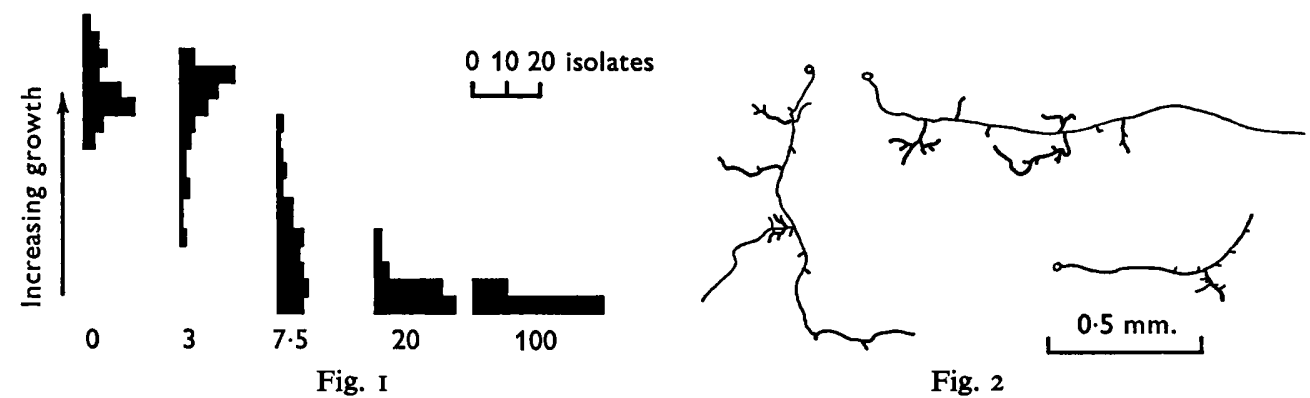

Fig. 2

Fig. I. Effect of streptomycin (concentrations $0,3,7 \cdot 5,20$, $100 \mu \mathrm{g} . / \mathrm{ml}$.) on growth of wildtype Phytophthora cactorum. Histograms show variation in response of 50 single zoospore isolates to each concentration. Vertical axis represents light absorption by colonies after growth from similar inocula for equal times, in grouped arbitrary units.

Fig. 2. Extent of growth in 2 days from wild-type zoospores of Phytophthora cactorum on medium containing streptomycin $20 \mu \mathrm{g}$. $/ \mathrm{ml}$.

Two isolates did not sporulate well and were discarded at stage (ii).

The results with the remaining six isolates were as follows:

(a) With three isolates, all twelve zoospore cultures grew well on all concentrations of streptomycin and on drug-free medium. These strains were uniformly resistant.

(b) With one isolate, the twelve zoospore cultures showed differences in tolerance and this occurred independently of whether they had been germinated on medium with or without streptomycin.

(c) With one isolate, all twelve zoospore cultures were uniformly sensitive, like the wild type. Apparently tolerance to streptomycin was only temporary and was lost during growth on drug-free medium.

(d) One isolate was streptomycin-dependent, and did not grow on the pea meal agar [stage (i)] unless this was supplemented with streptomycin. The I2 single zoospore cultures of this isolate, six of which were rescued from streptomycin-free medium before growth ceased, were all uniformly dependent.

One of the resistant isolates and the dependent isolate were studied in detail; they were designated $S r$ and $S d$, respectively.

\section{Inheritance of resistance and dependence}

In asexual progeny. The growth of 50 single zoospore cultures of $S r$ and $S d$ on various concentrations of streptomycin was examined. Zoospores of $S d$ had to be grown on medium containing streptomycin, since on SMA growth ceased soon after production of a short branched germ tube (and it was not resumed when suitable amounts of streptomycin were added). To prevent streptomycin being carried over to drug-free medium in the inocula of $S d$, zoospores were grown into small colonies on SMA + streptomycin $100 \mu \mathrm{g} . / \mathrm{ml}$. and disc inocula from these were transferred to SMA where sufficient growth took place to provide drug-free inocula for testing on various 
drug concentrations. Zoospores of $S r$ were germinated directly on SMA. Inocula from 50 single-zoospore isolates of each strain were grown on SMA + streptomycin o, IO, 100 and $1000 \mu \mathrm{g}$. $/ \mathrm{ml}$. and the growth measured photometrically. Results are shown in Figs. 3 and 4.

In sexual progeny. The results obtained with the above 50 zoospores were designed to serve as a standard of variation to test whether there was any segregation for drug resistance in the sexual progenies of these strains. Oospores resulting from selfing were germinated on plain agar and transferred to SMA and SMA+streptomycin

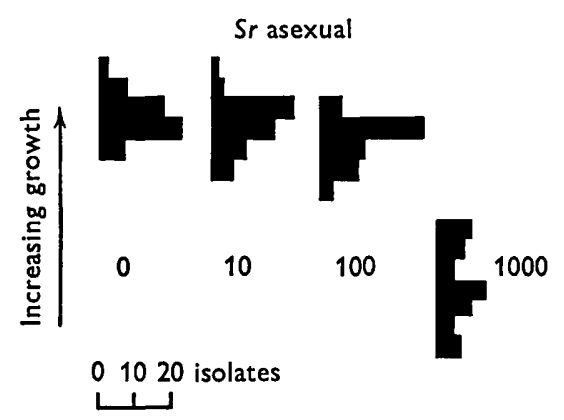

Fig. 3

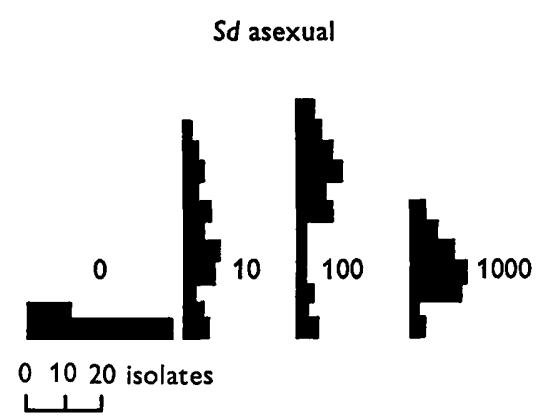

Fig. 4

Figs. 3, 4. Comparison of growth responses of asexual progenies of Phytophthora cactorum strains $S r$ and $S d$ to streptomycin o, 10, 100 and $1000 \mu \mathrm{g} . / \mathrm{ml}$. Fig. 3. Variation in growth of 50 single zoospore isolates of $S r$. Fig. 4. Fifty single zoospore isolates of $S d$. Vertical axis represents light absorption by colonies grown in any one experiment from similar inocula for equal times; light absorption values in grouped arbitrary units.

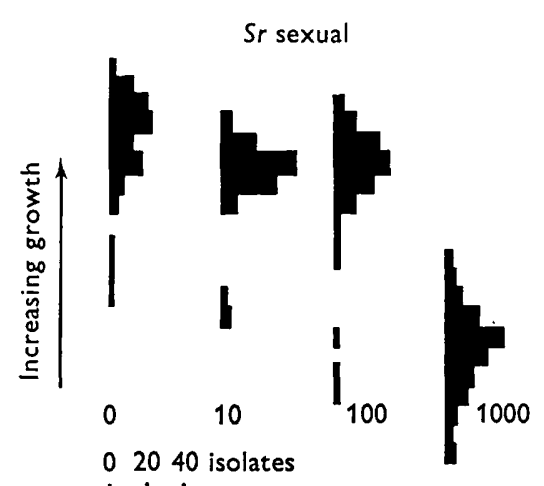

Fig. 5

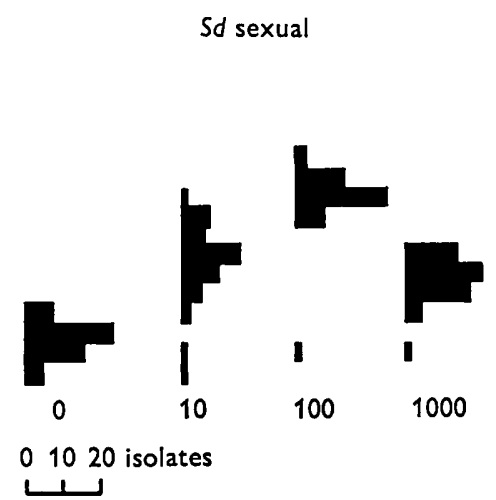

Fig. 6

Figs. 5, 6. Comparison of growth responses of selfed sexual progenies of Phytophthora cactorum strains $S r$ and $S d$ to streptomycin o, 10, 100 and $1000 \mu \mathrm{g} . / \mathrm{ml}$. Fig. 5. One hundred single oospore isolates of $S r$. Fig. 6. Fifty single oospore isolates of $S d$. Vertical axis as in Figs. 3 and 4.

I $00 \mu \mathrm{g} . / \mathrm{ml}$. Colonies from $S r$ oospores developed on both media, but growth from $S d$ oospores occurred only on SMA + streptomycin. Drug-free inocula were prepared from 50 germinated $S d$ oospores and $100 S r$ oospores in the same way as for zoospores and tested on the same four concentrations of streptomycin. The results of measurements of growth, made photometrically, are shown in Figs. 5 and 6. 
The curves showing the variation in growth at each concentration are essentially unimodal; there is no evidence of more than one kind of response to streptomycin in either of these progenies. The response is essentially the same as that of the zoospore progenies. (In a few cases the inoculum did not 'take' quickly but these developed later. This also occurred with wild type.) Thus no segregation occurred during sexual reproduction in either strain.

\section{Reversion of streptomycin-dependence to resistance}

When plates of SMA were inoculated centrally with a disc cut from a colony of $S d$ growing on SMA+streptomycin $100 \mu \mathrm{g} . / \mathrm{ml}$., initial growth compared favourably with that made by similar discs placed on medium with streptomycin $100 \mu \mathrm{g}$. $/ \mathrm{ml}$., but presumably with the exhaustion of the streptomycin in the inoculum, growth slowed down and eventually stopped. With an inoculum disc $4 \mathrm{~mm}$. diam. the colonies attained a diameter of approximately $40 \mathrm{~mm}$. However, some 20 days after the initial inoculation, sectors were observed to arise from the edge of such colonies. These non-dependent sectors appeared in one case in all of 6 plates.

It was further found that when zoospores from $S d$ cultures were added to flasks of liquid standard medium without streptomycin, non-dependent colonies appeared with a frequency of about $3 \times 10^{-5}$ per zoospore.

These reverted strains, which could grow in the absence of streptomycin, could still tolerate streptomycin at $1000 \mu \mathrm{g}$. $/ \mathrm{ml}$. The change was from dependence to resistance.

\section{Morphological change in the resistance strains}

Spontaneous change in colony morphology is well known in Phytophthora (Leonian, 1925; Stamps, 1953). In the wild-type strain used here, sectors of different morphology were observed in occasional colonies, and in some platings of zoospores a few variants were noted, but this parent strain is comparatively stable. In striking contrast were the variety of morphological types and the frequency of their occurrence in some of the streptomycin-resistant isolates.

The morphological variation in the streptomycin-dependent strain, $S d$, growing on SMA + streptomycin $100 \mu \mathrm{g} . / \mathrm{ml}$., was particularly distinct, and was studied in detail. Three morphological types occurred in the first plating of zoospores and were distinguished as follows (Pl. I, fig. I $a$, I $b$ ): (i) plumulose, $p$, with the same morphology as wild type; (ii) knotted, $k$, which differed from $p$ in its rate of increase in colony diameter (on SMA + streptomycin it grew twice as fast as $p$ ), in its more diffuse growth (with long leading hyphae and short knotted laterals) and in its lack of aerial mycelium: growth as measured photometrically, however, did not differ from $p$; (iii) regular, $r$, with growth rate similar to $p$, but with a more even edge to the colony and with much more dense aerial mycelium.

On plating zoospores from each of these types, $k$ and $r$ were found to be stable, but $p$ gave $33-40 \% k$ segregants. Plumulose $(p)$ colonies sometimes showed $k$ sectors. Later it was found that $k$ colonies which had risen from $p$ gave a small proportion of $p$ in their asexual progeny.

The nature of this variation between $p$ and $k$ was investigated further by comparing the proportions of $p$ and $k$ type colonies in asexual progeny of different single zoospores. The amount of vegetative growth was kept to a minimum by using the small discs of medium to produce zoospores. One disc was inoculated with mycelium from a 
single zoospore colony of $p$ type, and from this culture zoospores were obtained and plated on SMA + streptomycin. After incubation for $6 \mathrm{hr}, 60$ germinated spores were picked off on to discs. In addition, 6 discs were inoculated with a mass of mycelium from the original disc. Zoospores were obtained from most of the disc cultures and plated out, and for comparison, platings were made of zoospores from the six mycelial cultures. The proportion of $k$ type was determined in each of these progenies, which numbered from I4 to 272 , with an average of about I4O.

The results are shown in the histogram (Fig. 7). There were three kinds of progenies from single zoospores: (i) predominantly $p$, but including up to $6 \% k$ (25 progenies) (Pl. I, fig. 2); (ii) predominantly $k$, but including up to $5 \% p$ (22 progenies) (Pl. I, fig. 3); (iii) intermediate types (2 progenies) with 30 and $33 \% k$, which resembled the progeny of the mycelial mass cultures.

Table I. Effect of zoospore size on the proportion of $k$ type segregants from Phytophthora cactorum morphological type $p$

\begin{tabular}{|c|c|c|}
\hline $\begin{array}{c}\text { Zoospore } \\
\text { diameter }(\mu)\end{array}$ & $\begin{array}{c}\text { No. of } \\
\text { colonies }\end{array}$ & $\begin{array}{l}\text { Percentage } \\
k \text { segregants }\end{array}$ \\
\hline$>20$ & 39 & I3 \\
\hline $14-20$ & 40 & 45 \\
\hline$<\mathrm{I} 4$ & 40 & 70 \\
\hline
\end{tabular}

Table 2. Effect of storage at $3^{\circ}$ on the incidence of $k$ type morphologies in the asexual progeny of Phytophthora cactorum $p$ cultures

\begin{tabular}{|c|c|c|c|c|}
\hline \multicolumn{5}{|c|}{ Days at $3^{\circ}$} \\
\hline \multicolumn{5}{|c|}{${ }^{4}$ Percentage $k$ phenotypes* } \\
\hline $50 \cdot 6$ & $55 \cdot 4$ & $59 \cdot 2$ & $69 \cdot 9$ & $6 r \cdot 7$ \\
\hline \multicolumn{5}{|c|}{ Analysis of variance $\dagger$} \\
\hline & & & & Mean square \\
\hline & \multicolumn{4}{|c|}{ Between times } \\
\hline & gression & & & $122 \cdot 35 \ddagger$ \\
\hline & & & & $62 \cdot 32$ \\
\hline $\operatorname{Re}$ & & & & $18 \cdot 78$ \\
\hline
\end{tabular}

* Means of three replicates. Percentages based on counts of from 107 to 232 colonies.

$\dagger$ Percentages transformed to angles.

$¥$ Significant at $5 \%$ level.

These results were obtained with the streptomycin-dependent strain growing on SMA + streptomycin $100 \mu \mathrm{g}$./ml. When later reverted non-dependent resistant strains were obtained (see above), it was shown that the observed segregation was inherent in the fungus and did not require the continued presence of streptomycin in the medium.

The segregation ratio observed does however depend on the size of the zoospores. When, instead of pea meal agar, SMA with added cholesterol $(30 \mu \mathrm{g}$. $/ \mathrm{ml}$.) was used as the pre-sporulation medium, zoospores of various sizes were obtained, the larger ones being multiflagellate and multinucleate. Such a suspension was obtained from a $p$ culture and plated on SMA+streptomycin. After $6 \mathrm{hr}$ single germinated zoospores were 
isolated and classified in three groups according to size. The proportion of $k$ segregants in each class was determined after the zoospores had grown for $72 \mathrm{hr}$ on SMA+ streptomycin; the results are shown in Table $\mathrm{I}$.

Change from $p$ to $k$ was also found to occur during storage of cultures under liquid paraffin. The effect of storage in the cold was studied in the following experiment. Pea-meal agar discs were inoculated with a $p$ strain and incubated for $72 \mathrm{hr}$. All except three discs were then transferred to $3^{\circ}$; these three discs were transferred immediately to non-nutrient agar to produce three separate zoospore suspensions, which were plated, and after 72-hr. incubation the colonies were scored for morphological type. Three discs were removed from storage at intervals, zoospores produced, and the percentage of different morphological types in the resulting colonies scored. The results (Table 2) show a significant change in the incidence of $k$ types with time of storage.

Oospores from both $p$ and $k$ cultures were germinated. As in the asexual progenies, a proportion of $k$ segregants appeared in the sexual progeny of $p$, and a proportion of $p$ segregants appeared in the sexual progeny of $k$. The asexual progeny of two single oospore cultures of $p$ phenotype both continued to segregate for $k$. The ability to segregate is not lost during sexual reproduction.

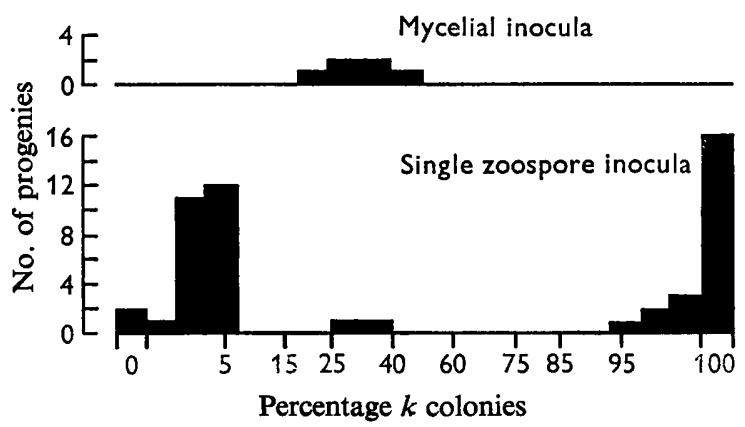

Fig. 7

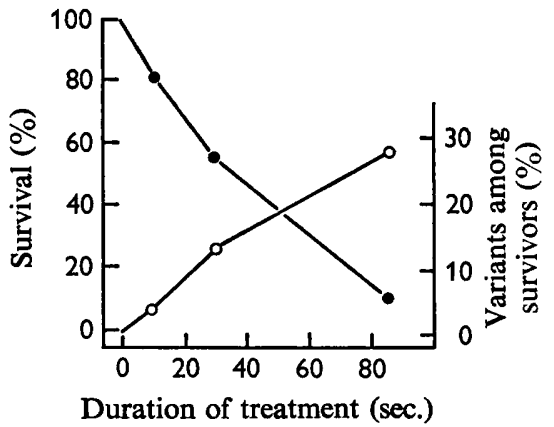

Fig. 8

Fig. 7. Segregation for morphological types $p$ and $k$ in asexual progeny of Phytophthora cactorum. Upper histogram, distribution of frequency of $k$ types in progeny of mass mycelial cultures. Lower histograms, distribution of frequency of $k$ types in progeny of single zoospore cultures. Progenies with 0 and $100 \% k$ shown separately, those with other values grouped in arbitrary equa 1 intervals after transformation of percentages to angles.

Fig. 8. Survival of zoospores of Phytophthora cactorum $\mathrm{Sr}$ after treatment with streptomycin (1000 $\mu \mathrm{g} . / \mathrm{ml}$.) [-O], and incidence of morphological variants among the survivors [O-O].

\section{The induction of morphological change by streptomycin}

The question arises as to whether the morphological variants observed in some of the streptomycin-resistant strains were the result of mutagenic action by streptomycin. This was examined by treating zoospores with a high concentration of streptomycin. Since wild-type zoospores are inhibited by streptomycin $20 \mu \mathrm{g} . / \mathrm{ml}$., the resistant strain, $S r$, was used. This has wild type morphology, is comparatively stable and the spores withstand streptomycin $1000 \mu \mathrm{g} . / \mathrm{ml}$.

A zoospore suspension containing streptomycin $1000 \mu \mathrm{g} . / \mathrm{ml}$. was prepared by adding $\mathrm{I} \mathrm{ml}$. of the sucrose-salt solution containing $10 \mathrm{mg}$. streptomycin to $9 \mathrm{ml}$. of zoospore suspension (about $\mathrm{I} \cdot 5 \times 10^{4}$ zoospores $/ \mathrm{ml}$.); $\mathrm{I} \mathrm{ml}$. of sucrose-salt solution 
was added to $9 \mathrm{ml}$. of control suspension. Samples were taken from the treated suspension after Io, 30 and $85 \mathrm{~min}$., suitably diluted and then plated. One plating of the control suspension was made. Streptomycin was added to the media in such quantities as to ensure that after plating the zoospores, all Petri dishes contained equal amounts of streptomycin (about $\mathrm{I} \cdot 5 \mu \mathrm{g}$. $/ \mathrm{ml}$ ). The number of variant colonies in the survivors was scored after $72 \mathrm{hr}$, and is shown in Fig. 8. Some of the morphological variants produced by this treatment are shown in Pl. I, fig. 5, and the control plating in Pl. I, fig. 4.

On subculture of a number of these variants to SMA, all reverted to wild-type morphology, and the zoospore progeny of I 9 variant colonies were also like wild type. Evidently the changes induced by streptomycin in this experiment were not hereditary, or were lost by selection of wild type.

Wild type was grown on SMA + streptomycin $5 \mu \mathrm{g} . / \mathrm{ml}$. and $S r$ on SMA $+500 \mu \mathrm{g}$./ $\mathrm{ml}$. for 5 weeks (cultures being transferred weekly). Zoospores were then produced and plated on SMA. The numbers of variants observed in these zoospore progenies did not exceed those in control platings from cultures grown on SMA for corresponding periods.

\section{DISCUSSION}

Streptomycin resistance and dependence are well known in bacteria. The higher fungi, however, are insensitive to streptomycin; the resistant and dependent strains we have obtained in the streptomycin-sensitive fungus Phytophthora cactorum are therefore of interest. The reversion of dependence to resistance is also noteworthy. Unfortunately there is no indication as to the nature of the changes which took place when these strains arose. The resistance and dependence are stable through asexual and sexual reproduction; no segregation was observed in selfed progeny. Resistance might be the result of a change either in a gene (in which case the implication is that the fungus is haploid) or in a cytoplasmic entity. Both genic and cytoplasmic streptomycin resistance are known in Chlamydomonas (Sager, 1954).

It was found that exposure to high concentrations of streptomycin for short periods induced phenotypic change, but in this experiment the change was only temporary. However, it cannot be ruled out that the hereditary morphology changes observed in many of the streptomycin-resistant strains were induced by the drug (cf. Sager \& Tsubo, 1962; Sager, 1962).

The heritable variation in morphology we observed has the following characteristics: differences between strains in the degree of variability; continuing segregation during vegetative growth and in the asexual progeny of single zoospore cultures; increased variability with storage; continuance of the ability to segregate following sexual reproduction. Many of these features were also described by Leonian (1925) and Stamps (I953). In the $S d$ strain, zoospores ordinarily have the potentiality to develop into colonies which have one or other of the alternative morphologies, $p$ and $k$, and which give nearly uniform progenies. This suggests that $p$ and $k$ are determined by particulate factors of which each zoospore has one. Large zoospores, which give a high proportion of both morphological types in their progeny, may have two or more of these factors. These factors are apparently mutable, but whether they are nuclear genes or unstable cytoplasmic entities cannot be determined in the absence of known nuclear gene markers. 
We wish to acknowledge with thanks the grant of a Science Research Council studentship to D.S.S.

\section{REFERENCES}

Buddenhagen, I. W. (1958). Induced mutations and variability in Phytophthora cactorum. Am. J. Bot. 45, 355.

Clarke, D. D. \& Robertson, N. F. (1966). Mutational studies on Phytophthora infestans. Eur. Potato J., 9, 208.

Leonian, L. H. (1925). Physiological studies on the genus Phytophthora. Am. J. Bot. 12, 444.

Middlekauff, J. E., Hino, S., YANG, S. P., Lindegren, G. \& Lindegren, C. C. (1957). Gene control of resistance vs. sensitivity to actidione in Saccharomyces. Genetics $\mathbf{4 2}, 66$.

Mullins, J. T. \& RAPER, J. R. (1965). Heterothallism in biflagellate aquatic fungi: preliminary genetic analysis. Science, N.Y. I50, II 74.

ROPER, J. A. \& KäFER, E. (1957). Acriflavine-resistant mutants of Aspergillus nidulans. J. gen. Microbiol. 16, 660.

SAGER, R. (1954). Mendelian and non-mendelian inheritance of streptomycin resistance in Chlamydomonas reinhardi. Proc. natn. Acad. Sci. U.S.A. 40, 356.

SAGER, R.(1962). Streptomycin as a mutagen for nonchromosomal genes. Proc. natn. Acad. Sci. U.S.A. $48,2018$.

SAGER, R. \& Tsubo, Y. (1962). Mutagenic effects of streptomycin in Chlamydomonas. Arch. Mikrobiol. 42, 159 .

SANSOME, E. (1961). Meiosis in the oogonium and antheridium of Pythium debaryanum Hesse. Nature, Lond. 191, 827.

Sansome, E. (1963). Meiosis in Pythium debaryanum Hesse and its significance in the life-history of the Biflagellatae. Trans. Br. mycol. Soc. 46, 63.

SAnsome, E. (1965). Meiosis in diploid and polyploid sex organs of Phytophthora and Achlya. Cytologia 30, I03.

Shaw, D. S. (1967). A method of obtaining single-oospore cultures of Phytophthora cactorum using live water snails. Phytopathology 57, 454.

StamPs, D. J. (1953). Variation in a strain of Phytophthora cactorum. Trans. Br. mycol. Soc. 36, 248.

WARR, J. R. \& ROPER, J. A. (1965). Resistance to various inhibitors in Aspergillus nidulans. J. gen. Microbiol. 40, 273.

\section{EXPLANATION OF PLATE}

Fig. I $a, \mathrm{I} b$. Plating of Phytophthora cactorum strain $S d$ showing the three morphological types, $p, k$ and $r$.

Fig. 2. Class (i) progeny of a single zoospore of $P$. cactorum $S d$; mainly type $p$ but showing four colonies of type $k$.

Fig. 3. Class (ii) progeny of a single zoospore of $P$. cactorum $S d$; mainly type $k$ but showing one colony of type $p$ (arrow).

Fig. 4. Plating of untreated zoospores of $P$. cactorum strain $S r$.

Fig. 5. Plating of zoospores of $P$. cactorum $S r$ treated with streptomycin (1000 $\mu \mathrm{g} . / \mathrm{ml}$.) for $85 \mathrm{~min}$. 
Journal of General Microbiology, Vol. 5I, No. I

Plate I
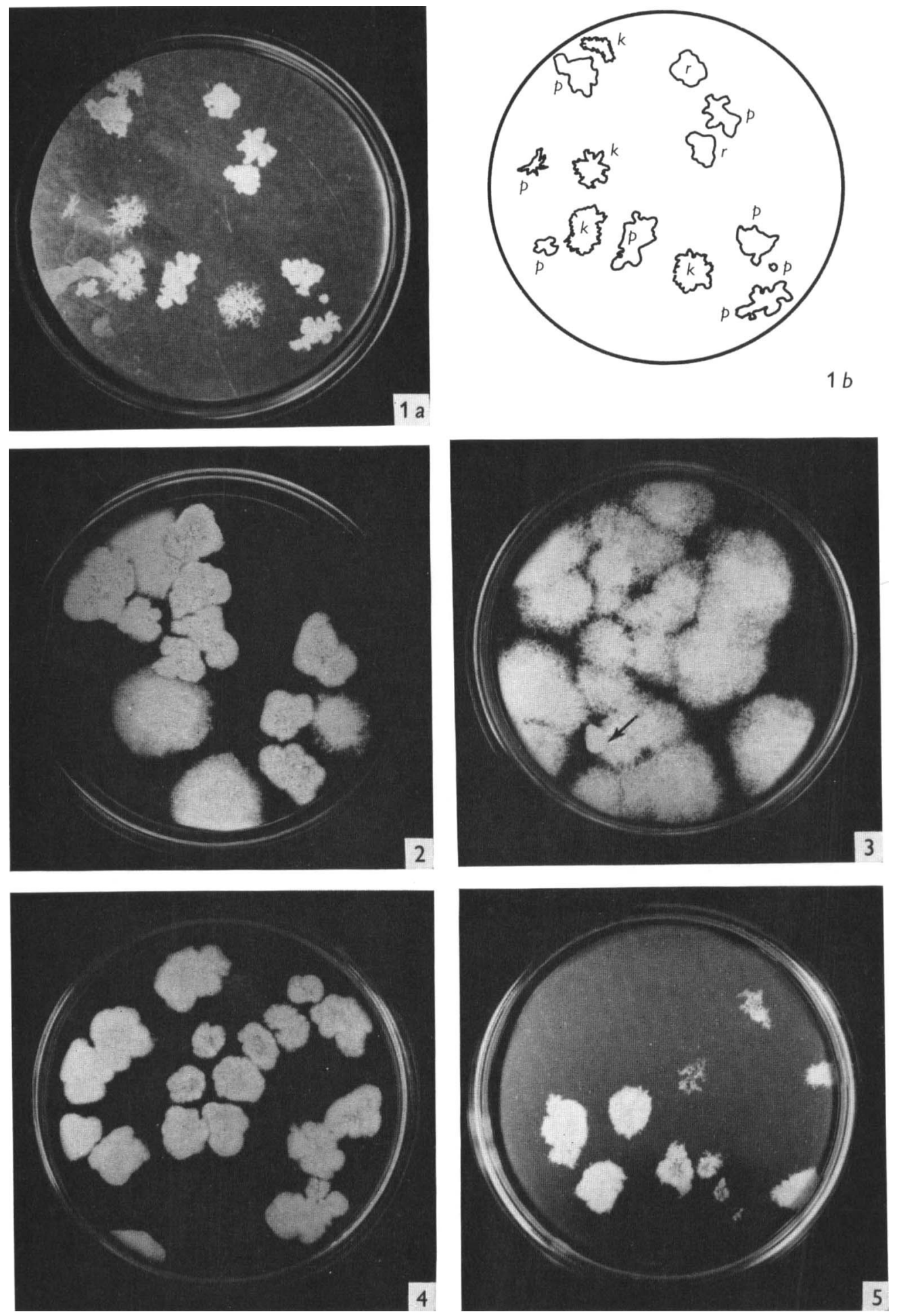

D. S. SHAW AND C. G. ELLIOTT

(Facing p. 84) 